\title{
Acknowledgement of Reviewers
}

We would like to acknowledge the support of numerous unsung international reviewers, the University of Technology Sydney Library and the immense support through the Pro Vice Chancellor and Vice President (Teaching and Learning, UTS) Dr. Alex Byrne. To all those reviewers who completed reviews, those who also kept on reviewing more papers and recommending other reviewers, thank you for making it possible for us to publish the AJICT in 2005 and 2006. If we have left any names out, it is inadvertent. Thank you.

\section{Assigned Reviewers}

Abderrahmane Lakas Ahmed Al-Ani

Amin Abbosh

Ann Blandford

Daniel L Silver

Dengsheng Zhang

Elaine Lawrence

Frank Jiang

Hangzai Luo

Jianping Fan

Jun Zheng

Mikko Vesisenaho

Muralikrishna Venkata Iyyanki

Pedro Jose Marron

Piotr Cholda

Sanjeev Sharma

Toshiyuki Amagasa

Vladmir Trajkovic

Yesufu K Thomas
Abdelkrim AMIRAT

A H Chan

Amoakoh Gyasi-Agyei

Artur Ziviani

Denis Deng

Donald Adjeroh

Fathi E. Abd El-Samie

Gerhard Bauch

Hongjun Xu

Johnson Agbinya

Michael Heywood

Maode Ma

Nahdia Tabassum

Peter Komisarczuk

Sachin Kumar

Saradha Arumugam

Thomas Joachim Odhiambo Afullo

Xiaojing Huang

Zoran S Bojkovic

\section{UTS ePress}

Dr Alex Byrne, Pro Vice Chancellor and Vice President (Teaching and Learning, University of Technology Sydney)

Ms Fides Datu Lawton (Acting Librarian, University of Technology Sydney - ePress)

Ms Janet Chelliah (Engineering Librarian, University of Technology Sydney)

Mr Richard Buggy (OJS Administrator, University of Technology Sydney) 